\title{
Correlations at large transverse momenta
}

\author{
A. P. Contogouris* \\ CERN, Geneva, Switzerland \\ and Laboratoire de Physique Théorique et Particules Elémentaires, Orsay, France \\ D. Schiff \\ Laboratoire de Physique Théorique et Particules Elémentaires, Orsay, France
}

(Received 25 September 1975)

\begin{abstract}
After a brief discussion of the failure of the constituent-interchange model (CIM) to account for recent experimental data on large- $q_{T}$ correlations, a hadronic-bremsstrahlung model is introduced. We analyze the structure of the large- $q_{T}$ events, we present calculations of the correlation coefficients for $p+p \rightarrow \pi^{0} \pi^{0}+X$ when the two $\pi^{0}$ s are produced at $90^{\circ}$ with respect to the colliding proton either in opposite directions or in the same direction, and we compare with the data and with the predictions of the CIM. Further predictions of the models and simple experimental tests are pointed out.
\end{abstract}

\section{INTRODUCTION-EXPERIMENTAL SITUATION}

The production of hadrons with large transverse momenta $q_{T}$ has been studied quite intensively during the last few years. ${ }^{1}$ Parton models, especially the constituent-interchange model (CIM), ${ }^{2}$ have been successful in accounting for the main features of the single-hadron $(h)$ spectra $(p+p$ $-h+X)$. Many of these features, however, have also been accounted for in models of different nature, in particular those based on hadronic bremsstrahlung. ${ }^{3,4}$

We believe that more crucial tests of the underlying physical mechanisms are provided by experiments on correlations and on the structure of the large $-q_{T}$ events. As we will show, models of different types lead to completely different predictions on these important features.

The main experimental facts on correlations at large $q_{T}$ can be summarized as follows:

(a) In $p+p-\pi^{0}+X$ consider a large $-q_{T}$ trigger at $90^{\circ}$ with respect to the CERN ISR beams and measure the associated mean charged multiplicity. In the hemisphere opposite to the $\pi^{0}$ this multiplicity increases almost linearly with $q_{T}$; and as a function of the polar and azimuthal angles it has a pronounced maximum in the direction opposite to the $\pi^{0}$. In the same hemisphere the multiplicity is also significant but smaller than in the opposite hemisphere (it varies little with $q_{T}$ ) and has a maximum in the direction of the $\pi^{0.5}$

(b) Consider $p+p \rightarrow \pi^{0}+\pi^{0}+X$ in the c.m. of $p p$ with the two $\pi^{\circ}$ 's produced at $90^{\circ}$ either (i) in opposite directions or (ii) in the same direction. In both cases the correlation coefficient (CC) increases fast and smoothly with the transverse momentum of either of the $\pi^{0}$ 's and attains large values $^{6}$ (see also Figs. 5 and 6 ). Notice that in case (ii) the $\mathrm{CC}$ is comparable (although probably somewhat smaller) to that in case (i).

(c) Consider $p+p \rightarrow \pi^{0}+h^{ \pm}+X$, where $h^{ \pm}$denotes the charged hadron and $\pi^{0}, h^{ \pm}$are produced in the c.m. at $90^{\circ}$, either (i) in opposite directions or (ii) in the same direction. With a $\pi^{\circ}$ trigger of $q_{T} \approx 3 \mathrm{GeV}$, at a given transverse momentum of $h^{ \pm}$, the probabilities for (i) and (ii) are comparable [although somewhat smaller in (ii)]. ${ }^{7}$

(d) Consider $p+p-\pi^{0}+X$ with the $\pi^{0}$ trigger of $q_{T} \geqslant 3 \mathrm{GeV}$ and at $\theta=55^{\circ}$ in the c.m. system. As a function of the azimuthal and polar angles $\phi$ and $\theta$ [or of $\phi$ and the pseudorapidity $\eta=\ln \tan (\theta / 2)]$, there is a strong enhancement of the mean charged multiplicity opposite to the $\pi^{0}$ trigger $\left(150^{\circ}<|\phi|<180^{\circ}, \eta \simeq-\eta_{\pi} 0\right)$, and there is also some enhancement in the $\pi^{0}$ direction $\left(0<|\phi|<30^{\circ}, \eta \simeq \eta_{\pi 0}\right)$. The over-all multiplicity opposite to the $\pi^{0}$ can be subdivided into one part which is symmetric about $\eta \sim 0$ (or about some small negative $\eta$ ) plus an excess of $\sim 0.6$ charged particles per event centered at $\eta \simeq-\eta_{\pi 0}$. The multiplicity in the $\pi^{0}$ direction can also be subdivided in a similar way (excess at $\left.\eta \simeq \eta_{\pi 0}\right){ }^{8,9}$

In the CIM, correlations have been studied for $p+p \rightarrow \pi+\pi+X$ when the two pions with large transverse momenta $q_{1 T}, q_{2 T}$ are produced at $90^{\circ}$ either (i) in opposite directions or (ii) in the same direction..$^{10}$ In case (i) the CIM is found to predict that the CC should show strong dips at the values $q_{1 T}=q_{2 T}$ (see Fig. 5 below); and in case (ii) it predicts that the $\mathrm{CC}$ should be much smaller (by 1-2 orders of magnitude) than in case (i) (see Fig. 6). Clearly these predictions contradict the experimental facts (b) and (c) above. In general, in the same direction with a large $-q_{T}$ hadron, the CIM predicts negligible probability for observing a second hadron of large $q_{T}$, or for observing ad- 
ditional hadrons in significant number. ${ }^{1,10}$ Roughly speaking, in this model there is an oppositeside "jet," but no "jet" alongside the trigger. This essential prediction is in contradiction with experimental facts (a)-(d) [(d) in particular], which appear to imply a two-"jet" structure of the large $-q_{T}$ events (albeit with the opposite-side jet considerably stronger and both jets quite broad).

The parton model of Berman, Bjorken, and Kogut (gluon exchange) and related extensions involving direct quark-quark interactions ${ }^{11}$ are two-jet models. However, they lead to inclusive distributions $\omega d \sigma / d^{3} q(p+p \rightarrow \pi+X) \propto q_{T}^{-4}$ in contradiction to established experimental facts $\left(\propto q_{T}{ }^{-8}\right)$.

The main part of this paper is an effort towards constructing a two-jet model which also gives $\omega d \sigma / d^{3} q(p+p-\pi+X) \sim q_{r}{ }^{-8}$. Its basis is the production of hadrons, e.g., in $p p$ collisions via hadronic bremsstrahlung. The effort is motivated by the fact that in a (classical) collision of two fast particles, when these undergo some deflection, two jets of photons emerge along the directions of the outgoing particles. If we assume a similar mechanism for hadron production at large angles ${ }^{3,4}$ we may expect that each of these jets will often contain one or more energetic hadrons (pions with large $q_{T}$ ); important correlations may then prevail even between two hadrons of large $q_{T}$ in the same direction.

The essentials of such mechanism have been developed in Refs. 3 and 4, where $\omega d \sigma / d^{3} q(p+p-\pi+X) \propto q_{T}^{-8}$ was obtained, together with good accounts of the data.

In Sec. II of the present paper we give a simpler derivation, within the bremsstrahlung model, of the inverse-power law [essentially $\omega\left(d \sigma / d^{3} q\right)$ $\propto q_{T}{ }^{-8}$ ] for inclusive meson production; our final form is also more complete than that of Ref. 4 in an important aspect. In Sec. III we analyze the structure of the large $-q_{T}$ events (jet structure) of the model. In Sec. IV we calculate the $\mathrm{CC}$ for two opposite-side mesons in our bremsstrahlung model and compare it to experimental data and to the predictions of the CIM. In Sec. $\mathrm{V}$ we calculate the CC for two mesons on the same side, and also compare it to data and to the CIM. Section VI contains a brief summary of the main predictions and experimental tests of bremsstrahlung together with our concluding remarks. Finally, the Appendix gives a derivation of some useful relation.

\section{SINGLE-PARTICLE DISTRIBUTIONS}

In our physical picture, hadronic collisions at small $q_{T}(\$ 1 \mathrm{GeV})$ may be governed by multipe- ripheral-type production (plus some small diffractive component), but at large $q_{T}$ hadronic bremsstrahlung takes over. ${ }^{4}$ The general mathematical expressions for the inclusive distributions can be formulated, for example, in $p p$ collisions when the emitted hadrons are vector mesons $(m)$, by an approach similar to photon bremsstrahlung. ${ }^{3}, 12,13$ We begin by following this formalism.

Consider emission of $n$ neutral vector mesons via the process $p_{1}+p_{2} \rightarrow p_{3}+p_{4}+n m$, where $p_{\alpha}$ denotes a proton with four-momentum $p_{\alpha}$. The differential cross section after proper summation over polarizations and spins is ${ }^{12}$

$$
\begin{aligned}
d \sigma_{n}= & \frac{\left|M_{0}\right|^{2}}{4 \pi^{2}} \frac{M^{4}}{E_{1} E_{2} v_{12}} \delta^{4}\left(p_{1}+p_{2}-p_{3}-p_{4}-\sum_{i=1}^{n} q_{i}\right) \\
& \times\left[\frac{g^{2}}{(2 \pi)^{3}}\right]^{n} \prod_{i=1} V\left(q_{i}\right) \cdot V\left(q_{i}\right) \frac{d^{3} q_{i}}{2 \omega_{i}} \frac{d^{3} p_{3}}{E_{3}} \frac{d^{3} p_{4}}{E_{4}},
\end{aligned}
$$

where $M$ is the proton mass, $E_{\alpha}$ the proton energies, $v_{12}$ the relative velocity of the incoming protons, $q_{i}, \omega_{i}$ are the momenta and energies of the mesons $m, g$ is the $m$-proton coupling constant; $M_{0}=M_{0}\left(p_{1}, p_{2} ; p_{3}, p_{4}\right)$ the spin-averaged amplitude for the off-shell "elastic" process $p_{1}+p_{2} \rightarrow p_{3}+p_{4}$, and ${ }^{14}$

$$
\begin{aligned}
V(q)= & \frac{2 p_{1}}{q^{2}-2 p_{1} \cdot q}+\frac{2 p_{2}}{q^{2}-2 p_{2} \cdot q}+\frac{2 p_{3}}{q^{2}+2 p_{3} \cdot q} \\
& +\frac{2 p_{4}}{q^{2}+2 p_{4} \cdot q} .
\end{aligned}
$$

The invariant inclusive cross section for singlemeson production $\left(p_{1}+p_{2} \rightarrow m+X\right)$ is

$$
\begin{aligned}
\omega \frac{d \sigma}{d^{3} q}= & \frac{M^{4}}{4 E_{1} E_{2} v_{12}} \\
& \times \int \frac{d^{3} p_{3}}{E_{3}} \int \frac{d^{3} p_{4}}{E_{4}} \int \frac{d^{4} x}{(2 \pi)^{4}} e^{i x \cdot(P-q)} e^{K(x)} \\
& \times \frac{\left|M_{0}\right|^{2}}{4 \pi^{2}} \frac{g^{2}}{2 \pi^{3}} V^{2}(q),
\end{aligned}
$$

where $P \equiv p_{1}+p_{2}-p_{3}-p_{4}$ and

$$
K(x)=\frac{g^{2}}{(2 \pi)^{3}} \int \frac{d^{3} q}{2 \omega} e^{-i q \cdot x} V^{2}(q) .
$$

We consider the meson $m$ to be produced at $90^{\circ}$ in the c.m. of the colliding protons $\left(q_{L}=0\right)$ and begin with values of the variable $x_{T}=2 q_{T} / \sqrt{s}$ away from $x_{T}=1$, specifically

$$
M^{2} \ll 4 q_{T}^{2} \ll s ;
$$

all the presently available ISR large $-q_{T}$ data satisfy this condition. To be able to derive tractable 
expressions for the inclusive distributions we follow $^{4}$ the method of Fried and Gaisser ${ }^{3,12}$ : In the $p p$ c.m. (where $E_{1}=E_{2}=\sqrt{s} / 2$ ) we introduce the elasticity $\xi=2 E_{3} / \sqrt{s}$ of the proton 3 and replace in (2.3):

$$
\begin{aligned}
& \int \frac{d^{4} x}{(2 \pi)^{4}} e^{i x \cdot(P-q)} e^{K(x)} \\
& \quad-\int d \xi \delta^{3}\left(\overrightarrow{\mathrm{p}}_{3}+\overrightarrow{\mathrm{p}}_{4}+\overrightarrow{\mathrm{q}}+\left\langle\sum \overrightarrow{\mathrm{q}}_{i}^{a}\right\rangle\right) \delta\left(E_{3}-\xi \frac{1}{2} \sqrt{s}\right) P(\xi) e^{K(0)} .
\end{aligned}
$$

$\boldsymbol{P}(\xi)=$ proton probability distribution which can be determined from data on $p_{1}+p_{2} \rightarrow p_{3}+X ;\left\langle\sum \overrightarrow{\mathrm{q}}_{i}^{a}\right\rangle$ is an average total c.m. momentum carried by the unobserved hadrons produced in association with the meson $m$. As we discuss in the Appendix (see also Refs. 3 and 4),

$$
\overrightarrow{\mathrm{q}}+\left\langle\sum \overrightarrow{\mathrm{q}}_{i}^{a}\right\rangle \simeq 0
$$

Replacing in (2.3) and using $E_{3}^{-1} d^{3} p_{3}=\left|\vec{p}_{3}\right| d \Omega_{3} d E_{3}$ we obtain

$$
\begin{aligned}
\omega \frac{d \sigma}{d^{3} q} \simeq & \frac{M^{4}}{s} \frac{g^{2}}{(2 \pi)^{3}} \\
& \times \int d \Omega_{3} \int d \xi \frac{\left|\overrightarrow{\mathrm{p}}_{3}\right|}{E_{4}} \frac{\left|M_{0}\right|^{2}}{4 \pi^{2}} V^{2}(q) P(\xi) e^{K(0)} .
\end{aligned}
$$

Throughout this paper we work with the leading term in the expressions of the inclusive mesondistributions. $\left|M_{0}\right|$ and $K(0)$ are smooth functions of the scalar products $p_{\alpha} \cdot p_{\beta}$ of the four-momenta of the nucleons, $\alpha, \beta=1,2,3,4$ (see Ref. 4 and below). Thus the leading contribution to (2.7) arises from the kinematic configuration that maximizes $V^{2}(q)$. Suppose that $p_{3}$ is the final nucleon that moves in the same hemisphere with the observed meson $m$ [Fig. 1(a)]. From (2.2) it easily follows that $V^{2}(q)=$ maximum when the denominator $q^{2}+2 q \cdot p_{3}=m^{2}+2 q \cdot p_{3}=$ minimum. For $|\vec{q}|^{2} \gg M^{2}$ [condition (2.4)] the configurations of importance in our bremsstrahlung formalism correspond to $\left|\overrightarrow{\mathrm{p}}_{3}\right|^{2} \gg M^{2}$ as well. ${ }^{4}$ Then the maximum of $V^{2}(q)$ corresponds to the final nucleon $p_{3}$ moving in the same direction with the meson $m\left(\hat{p}_{3} \simeq \hat{q} \equiv \vec{q} /|\vec{q}|\right)$, very much the same as in the classical bremsstrahlung of photons by fast-moving charged particles: Then, let $\left|\vec{p}_{3}\right|=\alpha q_{T}$. Because of the condition (2.6), $\overrightarrow{\mathrm{p}}_{4} \simeq-\overrightarrow{\mathrm{p}}_{3}$ and we easily find

$$
\begin{aligned}
& p_{1} \cdot q=p_{2} \cdot q \simeq \frac{1}{2} \sqrt{s} q_{T}, \quad p_{3} \cdot q \simeq \frac{1}{2}\left(\frac{M^{2}}{\alpha}+\alpha m^{2}\right), \\
& p_{4} \cdot q \simeq 2 \alpha q_{T}^{2}, \\
& p_{1} \cdot p_{3} \simeq p_{2} \cdot p_{3} \simeq \frac{\alpha}{2} \sqrt{s} q_{T}, \quad p_{3} \cdot p_{4} \simeq 2 \alpha^{2} q_{T}^{2} .
\end{aligned}
$$

Finally,

$$
V^{2}(q) \approx \frac{4 \alpha(1+\alpha) m^{2}}{\left[(1+\alpha) m^{2}+M^{2} / \alpha\right]^{2}} \equiv V^{2}(\alpha) .
$$

Thus for fixed $\alpha$ the leading term, denoted by $V^{2}(\alpha)$, is independent of $q_{T}$. This leading term arises by integrating $d \Omega_{3}=2 \pi \sin \theta_{3} d \theta_{3}$ near the direction $\hat{q}$ in a small angle of opening $\Delta \theta_{3} \sim m / q_{T}{ }^{4}$

As in Ref. 4 we may argue (essentially on the basis of elastic $p p$ scattering) that for large $p_{3 T}, p_{4 T}$

$$
\left|M_{0}\right| \propto \frac{\sqrt{s}}{\left(p_{3 T} p_{4 T}\right)^{4}},
$$

so that in the present case $\left|M_{0}\right| \propto \sqrt{s}\left(\alpha q_{T}\right)^{-8}$. On
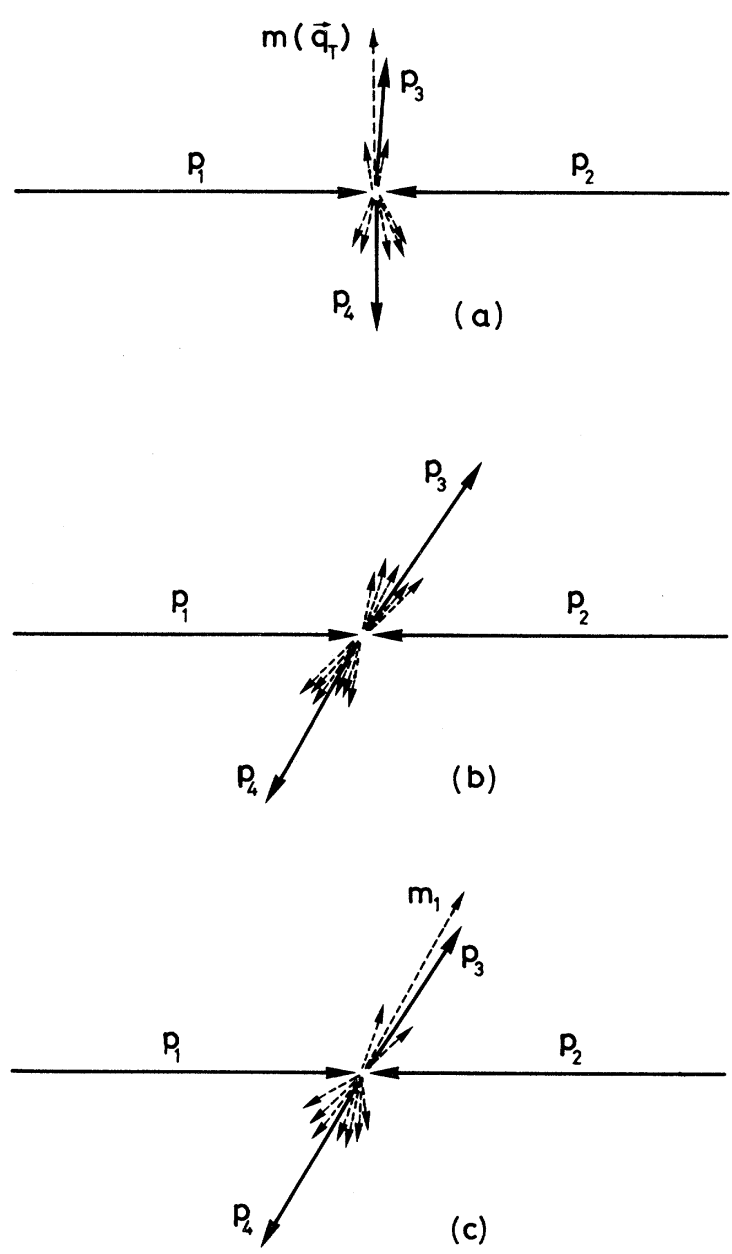

FIG. 1. (a) Kinematic configuration giving a leading contribution to $p+p \rightarrow m+X$ when the meson $m$ is produced at $\theta \simeq 90^{\circ}$ in the c.m. of the colliding protons. Solid lines: Baryons; dashed lines: mesons. (b) Typical large- $q_{T}$ event (jet structure) in bremsstrahlung. (c) Same as Fig. 1(a) but with the meson produced at $\theta<90^{\circ}$. 
the other hand, $K(0)$ has been calculated in detail ${ }^{4}$ and gives

$$
e^{K(0)} \simeq\left(q_{T} / m\right)^{G(g, \ln s)} .
$$

In particular, Ref. 4 obtained $G \simeq\left(g^{2} / \pi^{2}\right) \ln \left(s / 4 m^{2}\right)$, so that for $m \sim 1 \mathrm{GeV}, g^{2} / 4 \pi \approx 1$ (typical vector meson-nucleon coupling) and for ISR energies $G \approx 9-10 . .^{15}$ Replacing (2.9)-(2.11) in (2.7) and using $m \simeq 1$ we obtain

$$
\omega \frac{d \sigma}{d^{3} q} \propto \frac{1}{q_{T}^{17-G}} \int d \xi P(\xi) \frac{V^{2}(\alpha)}{\alpha^{16}} .
$$

As we mentioned, $P(\xi)$ can be determined from experimental information on $p+p \rightarrow p+X$, and present data can be roughly accounted for with $P(\xi) \sim 1 / \xi$ (Ref. 4) (for $x_{T}$ not too close to 1 ).

Then

$$
\omega \frac{d \sigma}{d^{3} q} \propto \frac{1}{q_{T}^{17-G}} \int_{\alpha_{0}}^{\infty} d \alpha \frac{V^{2}(\alpha)}{\alpha^{17}} .
$$

As discussed in Ref. 4, $\alpha_{0} \sim 1$. Thus we obtain an inverse power dependence on $q_{T}$; in fact, this is seen also from $(2.12)$ for a wide class of probability functions $P(\xi)$. From (2.9), $V^{2}(\alpha)$ varies with $\alpha$ slowly in contrast to the rapidly decreasing $\alpha^{-17}$, so that most of the contribution in the integral of (2.13) comes from $\alpha \simeq \alpha_{0}$. Thus

$$
\omega \frac{d \sigma}{d^{3} q} \propto V^{2}\left(\alpha_{0}\right) / q_{T}{ }^{17-G} .
$$

In Ref. 4 the first two terms in an expansion of $1 / q_{T}$ have been calculated; it is easy to see that the leading term $\left(\sim 1 / q_{T}{ }^{17-G}\right)$ is in agreement with the forms $(2.12)-(2.14)$. Thus with $G \approx 9-10$, $\omega\left(d \sigma / d^{3} q\right) \propto 1 / q_{T}{ }^{8}-1 / q_{T}{ }^{7}$.

The expressions (2.12)-(2.14) are expected to be valid when $x_{T}$ is small [when it satisfies (2.4)]; this is the case for all ISR data fitted in Ref. 4. When $x_{T} \rightarrow 1$, phase-space arguments imply $\omega\left(d \sigma / d^{3} q\right) \rightarrow 0$. Also the foregoing bremsstrahlung formalism can be rigorously justified in the softmeson approximation, ${ }^{3,12}$ which requires $x_{T}$ small.

In the limit $x_{T} \rightarrow 1$, to see the kind of modification needed we go back to the expression (2.3). Various arguments ${ }^{12,13}$ indicate that $x \approx 0$ gives a leading contribution to $K(x)$, so that

$$
\frac{d^{4} x}{(2 \pi)^{4}} e^{i x^{*}(P-q)} e^{K(x)} \sim e^{K(0)} \delta^{4}(P-q) .
$$

Writing $d^{3} p_{4} / E_{4}=2 d^{4} p_{4} \delta\left(p_{4}{ }^{2}-M^{2}\right) \theta\left(E_{4}\right)$ in $(2.3)$ and carrying the $d^{4} p_{4}$ integration, we obtain

$$
\begin{aligned}
\omega \frac{d \sigma}{d^{3} q} \propto \frac{1}{s} \int & \frac{d^{3} p_{3}}{E_{3}}\left|M_{0}\right|^{2} V^{2}(q) e^{K(0)} \delta^{4}\left(p_{4}^{2}-M^{2}\right) \\
& \times \theta\left(E_{4}\right)
\end{aligned}
$$

with $p_{4}=p_{1}+p_{2}-p_{3}-q$ everywhere in the integrand As before, the leading contribution arises from $\hat{p}_{3} \simeq \hat{q}$ and we integrate in a small cone $\Delta \Omega_{3}$ of opening $\Delta \theta_{3} \sim m / q_{T}$ in the direction $\hat{q}\left(\theta_{3} \simeq 90^{\circ}\right)$. As $x_{T} \rightarrow 1$ we find

$$
\omega \frac{d \sigma}{d^{3} q} \propto \frac{1}{s}\left|M_{0}\right|^{2} V^{2}(q) e^{K(0)} \int d \Omega_{3} \phi\left(q_{T}, s\right),
$$

where

$$
\phi\left(q_{T}, s\right) \equiv 4 \int p_{3} d E_{3} \delta\left(\left(p_{1}+p_{2}-p_{3}-q\right)^{2}-M^{2}\right) \theta\left(E_{4}\right) .
$$

In writing (2.17) we assume that as $x_{T} \rightarrow 1$ the product $\left|M_{0}\right|^{2} V^{2}(q) e^{K(0)}$ has a finite limit; this is justified in bremsstrahlung models..$^{3,42}$ Straightforward calculation gives for $q_{T}{ }^{2} \gg M^{2}$

$$
\phi\left(q_{T}, s\right) \simeq 1-\frac{2 q_{T}}{\sqrt{s}}=1-x_{T} .
$$

Thus as $x_{T} \rightarrow 1 \omega\left(d \sigma / d^{3} q\right) \propto 1-x_{T}$.

A complete theory of hadronic bremsstrahlung, covering the whole range $0<x_{T}<1$, is, unfortunately, lacking. Probably the simplest, intuitive way to combine $(2.14)$ with $(2.19)$ is to take

$$
\omega \frac{d \sigma}{d^{3} q}\left(q_{T}, q_{L}=0, s\right) \approx A \frac{1-x_{T}}{q_{T}^{17-G}},
$$

where $A$ is a constant $\left[\propto V^{2}\left(\alpha_{0}\right)\right]$ and for ISR ener gies $G \simeq 9-10$. The form $(2.20)$ is in many respects similar to that of parton models (for $x_{T}$ near 1$).^{2}$

Properly speaking, (2.20) has been obtained for $p+p \rightarrow m+X$, where $m=$ vector meson. To extend it to, for example, $p+p \rightarrow \pi^{ \pm}+X$, we may proceed in either of the following two ways: (i) Assume that the $\pi^{ \pm}$'s arise mainly from the decay of vector mesons; this assumption ${ }^{3,4}$ has recently received some consideration in connection with the production of large $-q_{T}$ leptons. ${ }^{16}$ Then for large $q_{T}$ the transverse momentum distribution of $\pi^{ \pm}$'s will be essentially the same as $(2.20) .{ }^{17}$ (ii) Assume a similar bremsstrahlung mechanism for direct pion production via an effective coupling, the details of which may be left unspecified. Plausibility arguments can be invoked leading to an inclusive cross section of the form (2.20).

\section{THE STRUCTURE OF LARGE- $\boldsymbol{q}_{T}$ EVENTS (JETS)}

We begin with the two-particle process

$$
p_{1}+p_{2} \rightarrow p_{3}+m+X
$$

and assume that in the c.m. of $p_{1} p_{2}$ the nucleon $p_{3}$ is produced at some fixed (large) angle with 
momentum $\left|\overrightarrow{\mathrm{p}}_{3}\right| \gg M$ [a proton trigger with large transverse momentum $p_{3 T}$, as in Fig. 1(b)]. We will determine in the bremsstrahlung model the directions at which the distribution of meson $m$ has a maximum.

From (2.3) we easily obtain the inclusive cross section for the process (3.1) when $m$ is a neutral vector meson:

$$
\begin{aligned}
E_{3} \omega \frac{d^{2} \sigma}{d^{3} p_{3} d^{3} q}= & \frac{M^{4}}{2 E_{1} E_{2} v_{12}} \\
& \times \int \frac{d^{3} p_{4}}{E_{4}} \int \frac{d^{4} x}{(2 \pi)^{4}} e^{i x \cdot(p-q)} e^{K(x)} \\
& \times \frac{\left|M_{0}\right|^{2}}{4 \pi^{2}} \frac{g^{2}}{(2 \pi)^{3}} V^{2}(q) .
\end{aligned}
$$

We again use the replacement (2.5) with the relation (2.6) and, carrying the $d^{3} p_{4}$ and $d \xi$ integrations, get

$E_{3} \omega \frac{d^{2} \sigma}{d^{3} p_{3} d^{3} q} \simeq \frac{4 M^{4}}{s^{3 / 2} E_{3}} \frac{\left|M_{0}\right|^{2}}{4 \pi^{2}} \frac{g^{2}}{(2 \pi)^{3}} V^{2}(q) e^{K(0)} P\left(\frac{2 E_{3}}{\sqrt{s}}\right)$.

As in Sec. II, $\left|M_{0}\right|$ and $K(0)$ are smooth functions of the scalars $p_{\alpha} \cdot p_{\beta}$ so that the maxima of (3.3) are determined from $V^{2}(q)$. Again the direction $\hat{q} \simeq \hat{p}_{3}$ provides a maximum [Fig. 1(b)]. Another direction that maximizes $V^{2}(q)$ corresponds to $\hat{q} \simeq \hat{p}_{4}$ so that $q^{2}+2 q \cdot p_{4}=m^{2}+2\left(\omega E_{4}-\overrightarrow{\mathrm{q}} \cdot \overrightarrow{\mathrm{p}}_{4}\right)$ $=$ minimum. In view of $(2.6), \hat{p}_{4} \simeq-\hat{p}_{3}$ so that the second direction is opposite to that of nucleon $p_{3}$. We may conclude that for large $\left|\vec{p}_{3}\right|$ there should be two "jets" of hadrons (mesons): one in the same direction with nucleon $p_{3}$ and one in the opposite direction [Fig. 1(b)]. Again the whole picture is very much the same as in classical bremsstrahlung of photons.

The form (2.2) of $V(q)$ implies that in the c.m. of the colliding protons $p_{1} p_{2}$ and also along the directions $\hat{p}_{1}$ and $\hat{p}_{2}=-\hat{p}_{1}$ there should be "jets" of mesons [not shown in Fig. 1(b)]. This prediction is typical of a number of models for hadron production at large transverse momentum. ${ }^{17}$

Consider also the reaction

$$
p+p \rightarrow m_{1}+m_{2}+X
$$

and assume that we fix the momentum $\overrightarrow{\mathrm{q}}_{1}$ of meson $m_{1}$ at some (large) angle and $\left|\vec{q}_{1}\right| \gg M$ [a meson trigger with large transverse momentum $q_{1 T}$, as in Fig. 1(c)]. The previous discussion implies that the kinematic configuragions that give a leading contribution to the reaction (3.4) correspond to one of the final (unobserved) nucleons, say $p_{3}$, emitted in about the same direction with $m_{1}\left(\hat{p}_{3} \approx \hat{q}_{1}\right)$. Then the distribution of $m_{2}$ should have a maximum in the direction $\hat{q}_{2} \approx-\hat{q}_{1}$ [a jet opposite to the trigger $m_{1}$, as in Fig. 1(c)]. In the same direction with the trigger $m_{1}\left(\hat{q}_{2} \approx \hat{q}_{1}\right)$ we also expect a maximum and a jet, but weaker than in the opposite direction (on account of general arguments of momentum conservation).

At the present transverse momenta of ISR triggers (2-7 GeV) (Refs. 5-9) we expect these jets to be quite broad [in $V(q)$, the minimum of the denominator, e.g., $q^{2}+2 q \cdot p_{4}$ $=m^{2}+2\left(\omega E_{4}-\overrightarrow{\mathrm{q}} \cdot \overrightarrow{\mathrm{p}}_{4}\right)$, will not be very sharp]. As the transverse momentum increases we expect the jets to become more and more collimated $\left(\omega E_{4}-\vec{q} \cdot \vec{p}_{4}\right.$ gets a sharper minimum in the direction $\hat{q} \simeq \hat{p}_{4}$ ). This again is much the same as in conventional photon bremsstrahlung.

One way to present the structure of the largetransverse-momentum events is via a plot of azimuthal angle $\phi$ versus the pseudorapidity $\eta=-\ln \tan (\theta / 2) .^{8}$ The angles $\theta$ and $\phi$ are defined in Fig. 2(a). We consider a $\pi^{0}$ trigger of $q_{T} \approx 3 \mathrm{GeV}$ and present in a qualitative manner the $\phi$ versus $\eta$ plots; first, that of our bremsstrahlung model [Figs. 2(b) and 2(c)] and second, that of the CIM [Figs. 3(b) and 3(c)], as studied in Ref. 10. For each of the models we present plots corresponding to two different triggering angles, $\theta_{\pi 0}=90^{\circ}$ and $\theta_{\pi 0}=55^{\circ}$ (generally, $\theta_{\pi 0}<90^{\circ}$ but large).

In the bremsstrahlung model when the trigger is at $90^{\circ}\left(\eta_{\pi^{0}}=0\right)$ the predicted structure is as follows [Fig. 2(b)]:

(1) a strong and broad jet opposite to the trigger (centered at $\phi=180^{\circ}$ and $\eta=\eta_{\pi 0}=0$ );

(2) a weaker jet alongside the trigger (centered at $\phi=0^{\circ}$ and $\left.\eta=\eta_{\pi 0}=0\right)$;

(3) A nucleon (more generally a baryon) opposite to the trigger, with a fairly large momentum $(z 1 \mathrm{GeV})$ [in Fig. 2(b) this is indicated by a circular shaded area];

(4) Another baryon alongside the trigger, again with momentum $\gtrsim 1 \mathrm{GeV}$ (other circular shaded area).

Again in the bremsstrahlung model, when the trigger is at $\theta_{\pi 0}=55^{\circ}$ (corresponding to $\left.\eta_{\pi 0}=0.65\right)$, we predict [Fig. 2(c)]:

(1) a strong and broad jet essentially in the direction opposite to the trigger (centered at $\phi=180^{\circ}$ and some $\eta$ between 0 and $\left.-\eta_{\pi 0}\right)^{18}$;

(2) a weaker jet essentially alongside the trigger (centered at $\phi=0^{\circ}$ and some $\eta$ between 0 and $\eta_{\pi 0^{18}}$

(3) a baryon opposite to the trigger $\left(\phi=180^{\circ}\right.$ and $\eta=-\eta_{\pi 0}$ ) with a fairly large momentum [circular shaded area in Fig. 2(c)] [in experiments with no particle identification ${ }^{8}, 9$ this baryon will represent an excess of one particle per event 


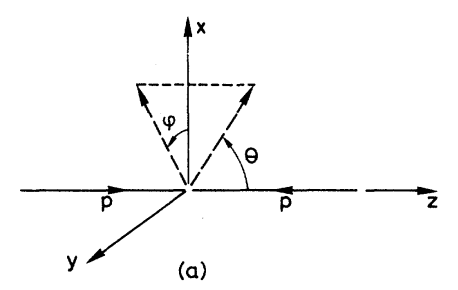

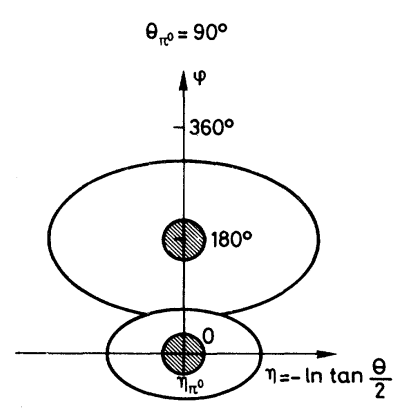

(b)

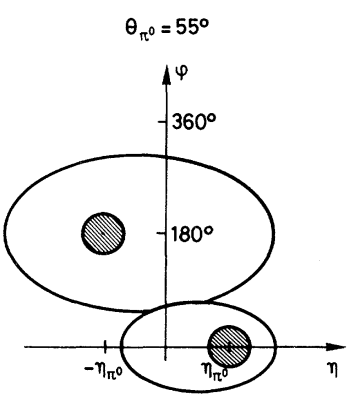

(c)
BREMSSTRAHLUNG MODEL

FIG. 2. (a) Definition of the angles $\theta$ and $\phi$. (b) Typical structure of a large $-q_{T}$ event ( $\phi$ versus $\eta$ plot) predicted in bremsstrahlung for $p+p \rightarrow \pi^{0}+X$ when $\theta_{\pi 0}=90^{\circ}$ (qualitatively). (c) Same as Fig. 2 (b) with $\theta_{\pi 0} \approx 55^{\circ}$.

(charged + neutral), and if only charged multiplicity is measured it will represent an excess of $\sim 0.6$ particles per event; clearly, this prediction is in qualitative agreement with the analysis of the ACHM collaboration ${ }^{8,9}$ ];

(4) Another baryon alongside the trigger $\left(\phi=0^{\circ}\right.$ and $\eta=\eta_{\pi 0}$ ) again with momentum $\approx 1 \mathrm{GeV}$ [other circular shaded area in Fig. 2(c)] (in an experiment without particle identification this will also represent a similar excess ${ }^{8,9}$ ).

Notice that a simple test of the bremsstrahlung model consists in determining the charge of the above excesses. ${ }^{19}$ Since we begin with two protons, we clearly predict that these excesses should be mainly positively charged. For example, if experiment gives equal percentages of positive and negative charge, the model will be in trouble.

We now turn to the qualitative structure of the large $-q_{T}$ events (jets) in the CIM. As we mentioned in the Introduction and have shown in Ref. 10 [see also Landshoff's repor $t^{1}$ ], this model predicts that there is an opposite-side jet, but

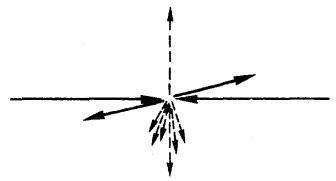

(a)

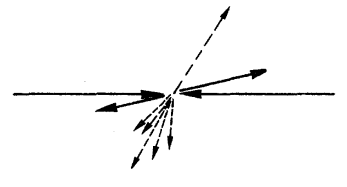

$\theta_{\pi^{0}}=90^{\circ}$

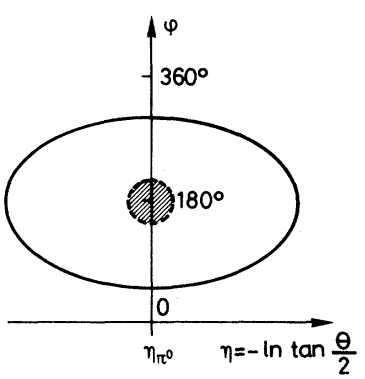

(b)

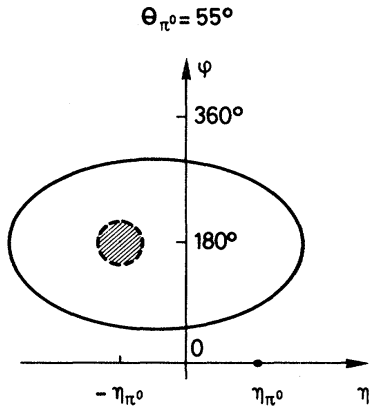

(c)

CONSTITUENT-INTERCHANGE MODEL

FIG. 3. (a) Typical large- $q_{T}$ events in CIM. Solid lines: baryons; dashed lines: mesons. (b) Typical structure of a large- $q_{T}$ event ( $\phi$ versus $\eta$ plot) predicted by CIM for $p+p \rightarrow \pi^{0}+X$ when $\theta_{\pi 0}=90^{\circ}$ (qualitatively). Notice that the final baryons are not shown in the figure (correspond to very large $|\eta|$ ). (c) Same as Fig. 3(b) with $\theta_{\pi 0} \approx 55^{\circ}$.

no jet on the same side with trigger. This is shown schematically in Fig. 3(a), for example for a $\pi^{\circ}$ trigger at $\theta_{\pi 0}=90^{\circ}$ and at $\theta_{\pi 0}<90^{\circ}$ (specifically $\theta_{\pi 0}=55^{\circ}$, i.e., $\left.\eta_{\pi 0}=0.65\right)$. In both cases a typical event is predicted to have the following characteristic features [Figs. 3(b) and 3(c)]:

(1) A strong jet in essentially the opposite side to the trigger; this is expected to be populated with mesons of low momentum $(<1 \mathrm{GeV})$;

(2) in addition, on the opposite side to the trigger, a few mesons of momentum $>1 \mathrm{GeV}$ [in Figs. 3(b) and 3(c) these fast mesons are indicated by a circular shaded area];

(3) two fast nucleons (with, say, $x_{L} \equiv 2 p_{L} / \sqrt{s}$ $z 0.5$ ) moving in the directions of the incident proton $\mathrm{s}^{20}$ [shown in Fig. 3(a), but not in Figs. $3(\mathrm{~b})$ and $3(\mathrm{c})$ ] (this prediction is typical of several parton models);

(4) no jet alongside the trigger (although 1-2 slow mesons may have a chance). 


\section{CORRELATION COEFFICIENT-OPPOSITE-SIDE HADRONS}

In this section we calculate the $\mathrm{CC}$ in the bremsstrahlung model for two large $-q_{T}$ hadrons produced in opposite sides, and make a comparison with the data of the CCR collaboration ${ }^{6}$ and with the predictions of the CIM.

We consider again the reaction (3.4) with the mesons $m_{1}, m_{2}$ produced at $90^{\circ}$ in the c.m. of the colliding protons in opposite directions [Fig. 4(a)]. As before, our bremsstrahlung formalism is more directly applicable if $m_{1}, m_{2}$ are vector mesons. From (2.1) we have

$$
\begin{aligned}
4 \omega_{1} \omega_{2} \frac{d^{2} \sigma_{n}}{d^{3} q_{1} d^{3} q_{2}}= & \frac{M^{4}}{E_{1} E_{2} v_{12}} \frac{d^{3} p_{3}}{E_{3}} \frac{d^{3} p_{4}}{E_{4}} \frac{\left|M_{0}\right|^{2}}{4 \pi^{2}} \frac{g^{4}}{(2 \pi)^{6}} V^{2}\left(q_{1}\right) V^{2}\left(q_{2}\right) \\
& \times \delta^{4}\left(P-q_{1}-q_{2}-\sum_{i=1}^{n} q_{i}\right)\left[\frac{g^{2}}{(2 \pi)^{3}}\right]^{n-2} \sum_{i=2}^{n} V^{2}\left(q_{i}\right) \frac{d^{3} q_{i}}{2 \omega_{i}}
\end{aligned}
$$

$\left(P=p_{1}+p_{2}-p_{3}-p_{4}\right)$. Following Ref. 12 we write the $\delta^{4}$ function in exponential representation:

$$
\begin{aligned}
4 \omega_{1} \omega_{2} \frac{d^{2} \sigma_{n}}{d^{3} q_{1} d^{3} q_{2}}= & \frac{M^{4}}{E_{1} E_{2} v_{12}} \frac{d^{3} p_{3}}{E_{3}} \frac{d^{3} p_{4}}{E_{4}} \frac{\left|M_{0}\right|^{2}}{4 \pi^{2}} \frac{g^{4}}{(2 \pi)^{6}} V^{2}\left(q_{1}\right) V^{2}\left(q_{2}\right) \\
& \times \int \frac{d^{4} x}{(2 \pi)^{4}} e^{i x \cdot\left(P-q_{1}-a_{2}\right)} \frac{1}{(n-2) !}\left[\frac{g^{2}}{(2 \pi)^{3}} \frac{d^{3} q}{2 \omega} V^{2}(q) e^{-i q \cdot x}\right]^{n-2},
\end{aligned}
$$

where the $n-2$ mesons of the final state are treated as identical. Summing over $n$ we obtain the following exact expression for the $m_{1}+m_{2}$ inclusive distribution:

$$
\begin{aligned}
4 \omega_{1} \omega_{2} \frac{d^{2} \sigma}{d^{3} q_{1} d^{3} q_{2}} & =\sum_{n=2}^{\infty} 4 \omega_{1} \omega_{2} \frac{d^{2} \sigma_{n}}{d^{3} q_{1} d^{3} q_{2}} \\
& =\frac{M^{4}}{2 E_{1} E_{2} v_{12}} \iint \frac{d^{3} p_{3}}{E_{3}} \frac{d^{3} p_{4}}{E_{4}} \frac{g^{4}}{(2 \pi)^{6}} \int \frac{d^{4} x}{(2 \pi)^{4}} e^{i x \cdot\left(P-q_{1}-q_{2}\right)} e^{K(x)} \frac{\left|M_{0}\right|^{2}}{4 \pi^{2}} V^{2}\left(q_{1}\right) V^{2}\left(q_{2}\right) .
\end{aligned}
$$

This expression can also be obtained directly from (2.3); we thought it worthwhile, however, to go through the above steps, which are typical for the derivation of formulas like (2.3) and (3.2).

We shall replace again

$$
\int \frac{d^{4} x}{(2 \pi)^{4}} e^{i x \cdot\left(P-a_{1}-a_{2}\right)} e^{K(x)}-\int_{0}^{1} d \xi \delta^{3}\left(\overrightarrow{\mathrm{p}}_{3}+\overrightarrow{\mathrm{p}}_{4}+\overrightarrow{\mathrm{q}}_{1}+\overrightarrow{\mathrm{q}}_{2}+\left\langle\sum \overrightarrow{\mathrm{q}}_{i}^{(a)}\right\rangle\right) \delta\left(E_{3}-\xi \frac{1}{2} \sqrt{s}\right) P(\xi) e^{K(0)},
$$

and because of the discussion in the Appendix

$$
\overrightarrow{\mathrm{q}}_{1}+\overrightarrow{\mathrm{q}}_{2}+\left\langle\sum \overrightarrow{\mathrm{q}}_{i}^{(a)}\right\rangle \approx 0
$$

By a procedure similar to that of Sec. II we obtain

$$
\omega_{1} \omega_{2} \frac{d^{2} \sigma}{d^{3} q_{1} d^{3} q_{2}} \simeq \frac{M^{4}}{2 s} \frac{g^{4}}{(2 \pi)^{6}} \int d \Omega_{3} \int d \xi \frac{\left|\overrightarrow{\mathrm{p}}_{3}\right|}{E_{4}} \frac{\left|M_{0}\right|^{2}}{4 \pi^{2}} V^{2}\left(q_{1}\right) V^{2}\left(q_{2}\right) P(\xi) e^{K(0)} \text {. }
$$

We proceed by keeping the leading term in $V^{2}\left(q_{1}\right), V^{2}\left(q_{2}\right)$. Let the mesons have momenta $q_{1 T}=\left|\overrightarrow{\mathrm{q}}_{1}\right| \gg m$ and $q_{2 T}=\left|\overrightarrow{\mathrm{q}}_{2}\right| \gg m$, as in Fig. 4(a). Previous discussions imply that the leading term in $V^{2}\left(q_{1}\right)$ and $V^{2}\left(q_{2}\right)$ arises when one of the final nucleons, say 3 , emerges in the same direction with the meson $m_{1}$ (i.e., $\hat{p}_{3} \simeq \hat{q}_{1}$ ); then the other final nucleon 4 emerges in the same direction with the meson $m_{2}\left(\hat{p}_{4} \approx \hat{q}_{2}=-\hat{q}_{1}\right)$. If we set $\left|\overrightarrow{\mathrm{p}}_{3}\right|=\alpha_{1} q_{1 T}$, the leading term of $V^{2}\left(q_{1}\right)$ will be

$$
V^{2}\left(q_{1}\right) \simeq V^{2}\left(\alpha_{1}\right)
$$

with $V^{2}\left(\alpha_{1}\right)$ given by (2.9). Likewise, with $\left|\overrightarrow{\mathrm{p}}_{4}\right|=\alpha_{2} q_{2 T}$, the leading term of $V^{2}\left(q_{2}\right)$ will be $\simeq V^{2}\left(\alpha_{2}\right)$. Again these leading terms arise by integrating $d \Omega_{3}=2 \pi \sin \theta_{3} d \theta_{3}$ near the directions $\hat{q}_{1}$ and $\hat{q}_{2}$, i.e., in two small angles of total opening $\Delta \theta_{3} \sim\left(m / q_{1 T}\right)+\left(m / q_{2 T}\right)$. The leading contribution is then

$$
\begin{aligned}
\omega_{1} \omega_{2} \frac{d^{2} \sigma}{d^{3} q_{1} d^{3} q_{2}} \propto & \frac{1}{s} \frac{q_{1 T}+q_{2 T}}{q_{1 T} q_{2 T}} \\
& \times \int d \xi\left|M_{0}\right|^{2} V^{2}\left(\alpha_{1}\right) V^{2}\left(\alpha_{2}\right) P(\xi) e^{K(0)} .
\end{aligned}
$$

$\left|M_{0}\right|$ will be taken as in $(2.10)$ so that here 


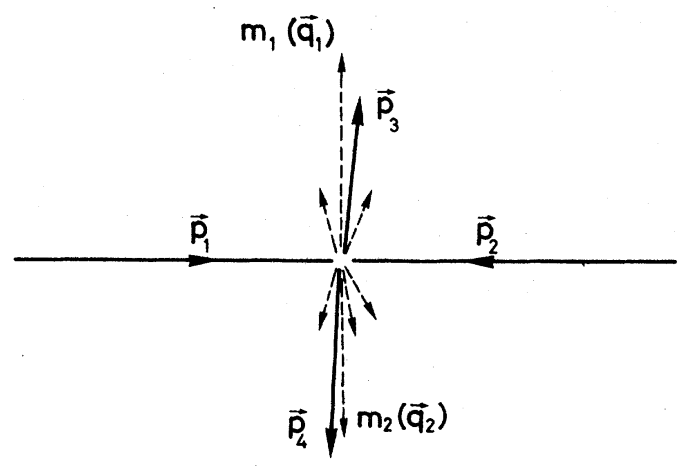

(a)

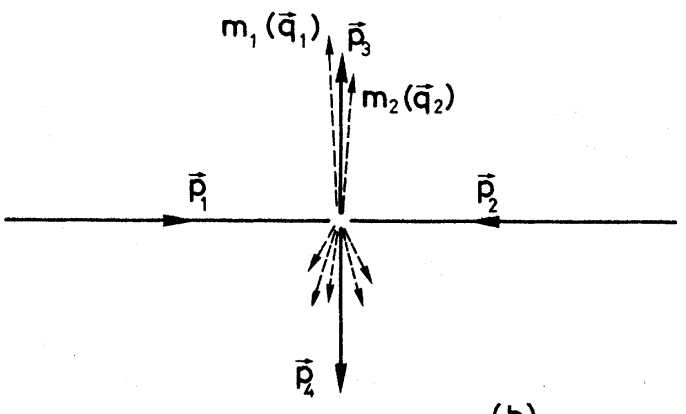

(b)

FIG. 4. (a) The reaction $p+p \rightarrow m_{1}+m_{2}+X$ when $m_{1}$, $m_{2}$ are produced at $90^{\circ}$ in opposite directions (bremsstrahlung). (b) As in Fig. 4 (a) with $m_{1}, m_{2}$ in the same direction.

$$
\left|M_{0}\right| \propto \frac{\sqrt{s}}{\left(\alpha_{1} \alpha_{2} q_{1 T} q_{2 T}\right)^{4}}
$$

and, as in Sec. II $P(\xi) \sim \xi^{-1}$. The calculation of Ref. 4 for $K(0)$ gives

$$
e^{K(0)} \simeq\left(\frac{q_{1} T q_{2} T}{m^{2}}\right)^{G / 2}
$$

where $G=G(g, \ln s)$ the same as in (2.11). Replacing in (4.6) we obtain $(m \simeq 1 \mathrm{GeV})$ :

$$
\begin{aligned}
\omega_{1} \omega_{2} \frac{d^{2} \sigma}{d^{3} q_{1} d^{3} q_{2}} & \simeq B_{1} \frac{q_{1 T}+q_{2 T}}{\left(q_{1 T} q_{2 T}\right)^{9-G / 2}} \\
& \times \int \frac{d \xi}{\xi} \frac{V^{2}\left(\alpha_{1}\right) V^{2}\left(\alpha_{2}\right)}{\left(\alpha_{1} \alpha_{2}\right)^{8}},
\end{aligned}
$$

where $B_{1}$ is a constant. We introduce the variable $\alpha=\left(\alpha_{1} \alpha_{2}\right)^{1 / 2}$ so that $\xi=2 E_{3} / \sqrt{s}=2 \alpha_{1} q_{1 T} / \sqrt{s}$ $=2 \alpha_{2} q_{2 T} / \sqrt{s}=2 \alpha\left(q_{1 T} q_{2 T}\right)^{1 / 2} / \sqrt{s}$ and

$$
\begin{aligned}
\omega_{1} \omega_{2} \frac{d^{2} \sigma}{d^{3} q_{1} d^{3} q_{2}} \simeq & B_{1} \frac{q_{1 T}+q_{2 T}}{\left(q_{1 T} q_{2 T}\right)^{9-G / 2}} \\
& \times \int \frac{d \alpha}{\alpha^{17}} V^{2}\left(\alpha\left(q_{2 T} / q_{1 T}\right)^{1 / 2}\right) \\
& \times V^{2}\left(\alpha\left(q_{1 T} / q_{2 T}\right)^{1 / 2}\right),
\end{aligned}
$$

where $\alpha_{0}$, the lower limit of the $\alpha$ integration, is of order 1.4 Clearly, the expression (4.7) is symmetric under $q_{1} \rightarrow q_{2}$, as the original (4.3). With $V^{2}\left(\alpha_{i}\right), i=1,2$, given by (2.9), the integral in (4.7) can be calculated exactly. Nevertheless, as in (2.14), we can further simplify by noting that $V^{2}\left(\alpha_{i}\right)$ are slowly varying functions of $\alpha_{i}$. Thus finally,

$$
\begin{aligned}
\omega_{1} \omega_{2} \frac{d^{2} \sigma}{d^{3} q_{1} d^{3} q_{2}} \approx & B \frac{q_{1 T}+q_{2 T}}{\left(q_{1 T} q_{2 T}\right)^{9-G / 2}} \\
& \times V^{2}\left(\alpha_{0}\left(q_{2 T} / q_{1 T}\right)^{1 / 2}\right) \\
& \times V^{2}\left(\alpha_{0}\left(q_{1 T} / q_{2 T}\right)^{1 / 2}\right),
\end{aligned}
$$

$B=B_{1} \alpha_{0}{ }^{-16} / 16=$ another constant.

The expressions (4.7), (4.8) are expected to hold in the range $M^{2} \ll 4 q_{i T}{ }^{2} \ll s$, i.e., $q_{i T}$ large but $x_{i T}$ not too close to 1 . To extend them to $x_{1 T} \rightarrow 1$ (or $x_{2 T} \rightarrow 1$ ) we proceed as in Sec. II. Starting from the exact expression (4.3) we first use

$$
\int \frac{d^{4} x}{(2 \pi)^{4}} e^{2 x \cdot\left(p-a_{1}-q_{2}\right)} e^{K(x)} \approx e^{K(0)} \delta^{4}\left(P-q_{1}-q_{2}\right) ;
$$

as before, the leading $V^{2}\left(q_{i}\right)$ correspond to $\hat{p}_{3} \simeq \hat{q}_{1}$ and $\hat{p}_{4} \simeq \hat{q}_{2}$; the limit $x_{1 T} \rightarrow 1$ implies $\left|\vec{p}_{3}\right| \rightarrow 0$; and assuming $\left|M_{0}\right|^{2} V^{2}\left(q_{1}\right) V^{2}\left(q_{2}\right) e^{K(0)} \rightarrow$ finite in this limit we obtain

$$
\begin{aligned}
\omega_{1} \omega_{2} \frac{d^{2} \sigma}{d^{3} q_{1} d^{3} q_{2}} \propto & \frac{1}{s}\left|M_{0}\right|^{2} V^{2}\left(q_{1}\right) V^{2}\left(q_{2}\right) e^{K(0)} \\
& \times \int d \Omega_{3} \phi\left(q_{1 T}, q_{2 T}, s\right),
\end{aligned}
$$

where

$$
\begin{aligned}
& \phi\left(q_{1 T}, q_{2 T}, s\right) \\
& \quad \equiv 4 \int\left|\overrightarrow{\mathrm{p}}_{3}\right| d E_{3} \delta\left(\left(p_{1}+p_{2}-p_{3}-q_{1}-q_{2}\right)^{2}-M^{2}\right) \theta\left(E_{4}\right) .
\end{aligned}
$$

Direct calculation in the limit $q_{1 T}{ }^{2},{q_{2 T}}^{2} \gg M^{2}$ gives

$$
\phi=\frac{2-x_{1 T}-x_{2 T}-\left|x_{1 T}-x_{2 T}\right|}{2-x_{1 T}-x_{2 T}+\left|x_{1 T}-x_{2 T}\right|} \equiv \phi_{-}\left(x_{1 T}, x_{2 T}\right) \text {. }
$$

Thus, for example, for $x_{2}=$ fixed $(<1)$ and $x_{1 T} \rightarrow 1$, $\omega_{1} \omega_{2} d^{2} \sigma / d^{3} q_{1} d^{3} q_{2} \sim 1-x_{1 T}$. Clearly, in Fig. 4(a) the mesons $m_{1}, m_{2}$ can be interchanged, resulting in $x_{1 T}-x_{2 T}$; this has been taken into account in writing (4.11). Finally, lacking a complete theory of hadronic bremsstrahlung, the simplest way to combine (4.8) and (4.11) is to take

$$
\begin{aligned}
\omega_{1} \omega_{2} \frac{d^{2} \sigma}{d^{3} q_{1} d^{3} q_{2}} \approx & B \frac{q_{1 T}+q_{2 T}}{\left(q_{1 T} q_{2 T}\right)^{9-G / 2}} V^{2}\left(\alpha_{0}\left(q_{2 T} / q_{1 T}\right)^{1 / 2}\right) \\
& \times V^{2}\left(\alpha_{0}\left(q_{1 T} / q_{2 T}\right)^{1 / 2}\right) \phi_{-}\left(x_{1 T}, x_{2 T}\right) .
\end{aligned}
$$


The CC for the two mesons $m_{1}, m_{2}$ (in $\left.p+p-m_{1}+m_{2}+X\right)$ can be defined as follows ${ }^{6}$ :

$$
R=\sigma_{\text {inel }} \omega_{1} \omega_{2} \frac{d^{2} \sigma}{d^{3} q_{1} d^{3} q_{2}} / \omega_{1} \frac{d \sigma}{d^{3} q_{1}} \omega_{2} \frac{d \sigma}{d^{3} q_{2}} .
$$

Using (2.20) and (4.12) and taking into account that in (2.20) $A \sim V^{2}\left(\alpha_{0}\right)$, we obtain the following expression for the CC $R_{-}$of $m_{1}, m_{2}$ produced at $90^{\circ}$ in opposite directions:

$$
\begin{aligned}
R_{-} \simeq & C\left(q_{1 T}+q_{2 T}\right)\left(q_{1 T} q_{2 T}\right)^{8-G / 2} \\
& \times \sigma_{\text {inel }} \frac{V^{2}\left(\alpha_{0}\left(q_{2 T} / q_{1 T}\right)^{1 / 2}\right) V^{2}\left(\alpha_{0}\left(q_{1 T} / q_{2 T}\right)^{1 / 2}\right)}{V^{4}\left(\alpha_{0}\right)} \\
& \times \frac{\phi_{-}\left(x_{1 T}, x_{2 T}\right)}{\left(1-x_{1 T}\right)\left(1-x_{2 T}\right)},
\end{aligned}
$$

where $C$ is a constant (depending on $\alpha_{0}$ ).

The last expression can be simplified by noting that the product $V^{2}\left(\alpha_{0}\left(q_{2 T} / q_{1 T}\right)^{1 / 2}\right) V^{2}\left(\alpha_{0}\left(q_{1 T} / q_{2 T}\right)^{1 / 2}\right)$ varies little with $q_{2 T} / q_{1 T}$ [insofar as $\left(q_{2 T} / q_{1 T}\right)^{1 / 2}$ does not become exceedingly small or large] and stays about equal to $V^{4}\left(\alpha_{0}\right)$. Thus finally

$$
\begin{aligned}
R_{-} \approx & C\left(q_{1 T}+q_{2 T}\right)\left(q_{1 T} q_{2 T}\right)^{8-G / 2} \\
& \times \sigma_{\text {inel }} \frac{\phi_{-}\left(x_{1 T}, x_{2 T}\right)}{\left(1-x_{1 T}\right)\left(1-x_{2 T}\right)} .
\end{aligned}
$$

The CCR collaboration has presented data for

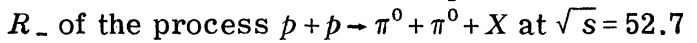
GeV. ${ }^{6}$ To compare with our results we use, as in Sec. II $g^{2} / 4 \pi=1$ and $m=1$, so that the exponent ${ }^{4}$ $G=\left(g^{2} / \pi^{2}\right) \ln \left(s / 4 m^{2}\right)=8.34$. The constant $C$ can, in principle, be determined; however, owing to the approximations anyway involved, we prefer to adjust its value at one point. Figure 5 presents comparison with the data of the more exact Eq. (4.14) (full lines); agreement is good.

It is of interest to present also some typical predictions of the CIM for $R_{-}$of $p+p \rightarrow \pi^{0}+\pi^{0}+X$ (Fig. 5, dashed lines), as calculated in Ref. 10 . The CIM predicts that at $q_{1 T} \approx q_{2 T}$ (or $x_{1 T} \approx x_{2 T}$, for fixed $s$ ) $R_{\text {_ }}$ should show strong dips (pronounced structure and strong variation, in general). ${ }^{10}$ This does not appear to be supported by experiment, which rather shows a smooth behav-

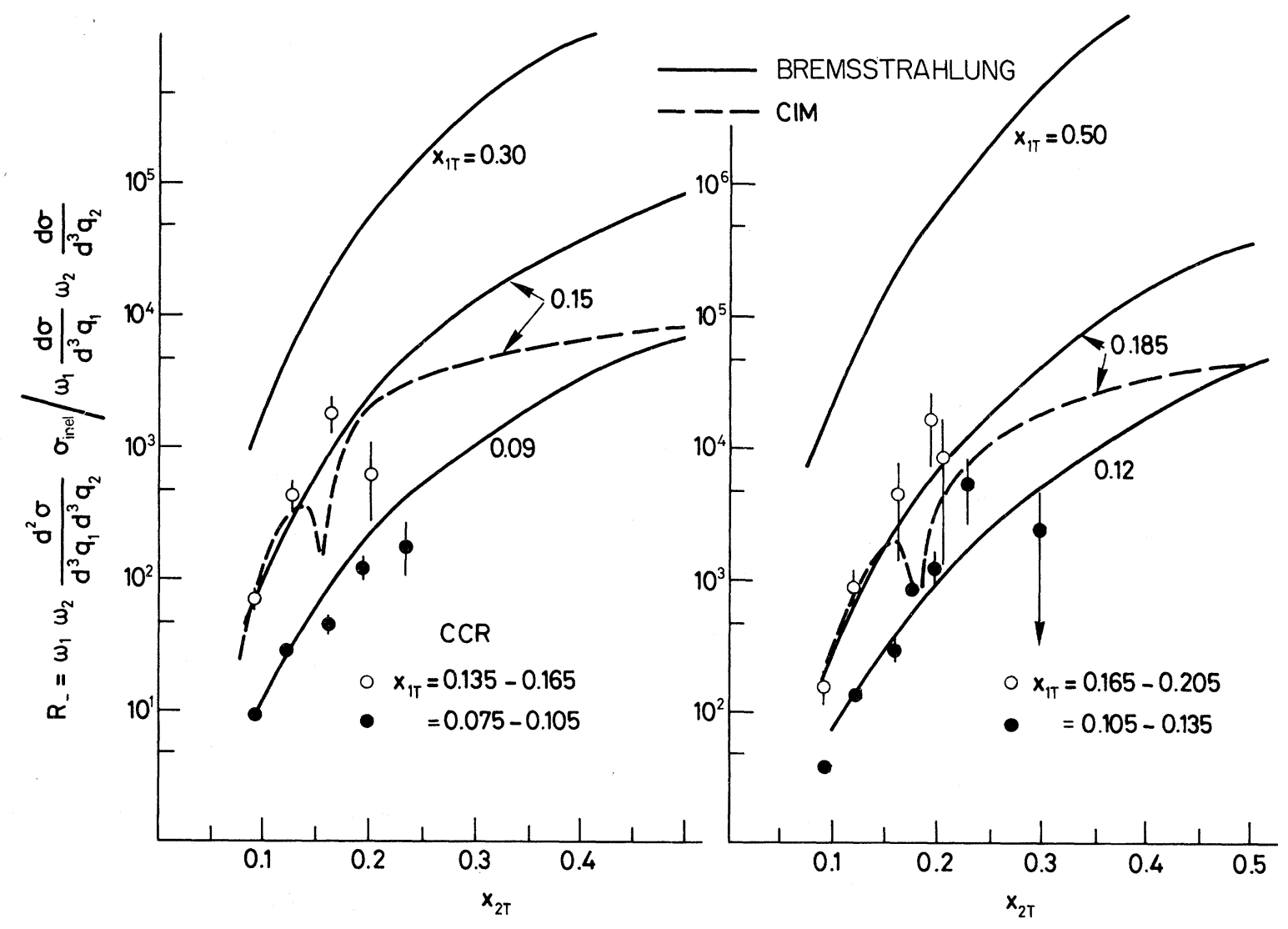

FIG. 5. The correlation coefficient $R_{-}$for two $\pi^{0} \mathrm{~s}$ at $90^{\circ}$ in the opposite direction. Predictions: solid lines are from bremsstrahlung; dashed lines are from CIM. Data from Ref. 6. 
ior of $R_{-}$. However, a definite conclusion should await for better data.

In contrast, a bremsstrahlung mechanism predicts that $R_{-}$continually increases with $x_{1 T}$ and $x_{2 T}$ also at $x_{1 T}=x_{2 T}$ (Fig. 5). Qualitatively, from (4.15), at fixed $\sqrt{s}[=52.7 \mathrm{GeV}$ (Ref. 6) $]$ and with $x_{1 T}, x_{2 T} \leqslant 0.25, R_{-}$is predicted to increase slightly faster than

$$
R_{-} \propto\left(x_{1 T}+x_{2 T}\right)\left(x_{1 T} x_{2 T}\right)^{3.83} .
$$

This appears to be supported by present data.

\section{CORRELATION COEFFICIENT-HADRONS ON SAME SIDE}

We turn now to a calculation of the CC for two mesons produced in the same direction, with large transverse momenta. This is one of the most critical quantities for large- $q_{T}$ hadron physics as most of the models fail to account for it. ${ }^{1,10}$

We consider again [reaction (3.6)]

$$
p+p \rightarrow m_{1}+m_{2}+X,
$$

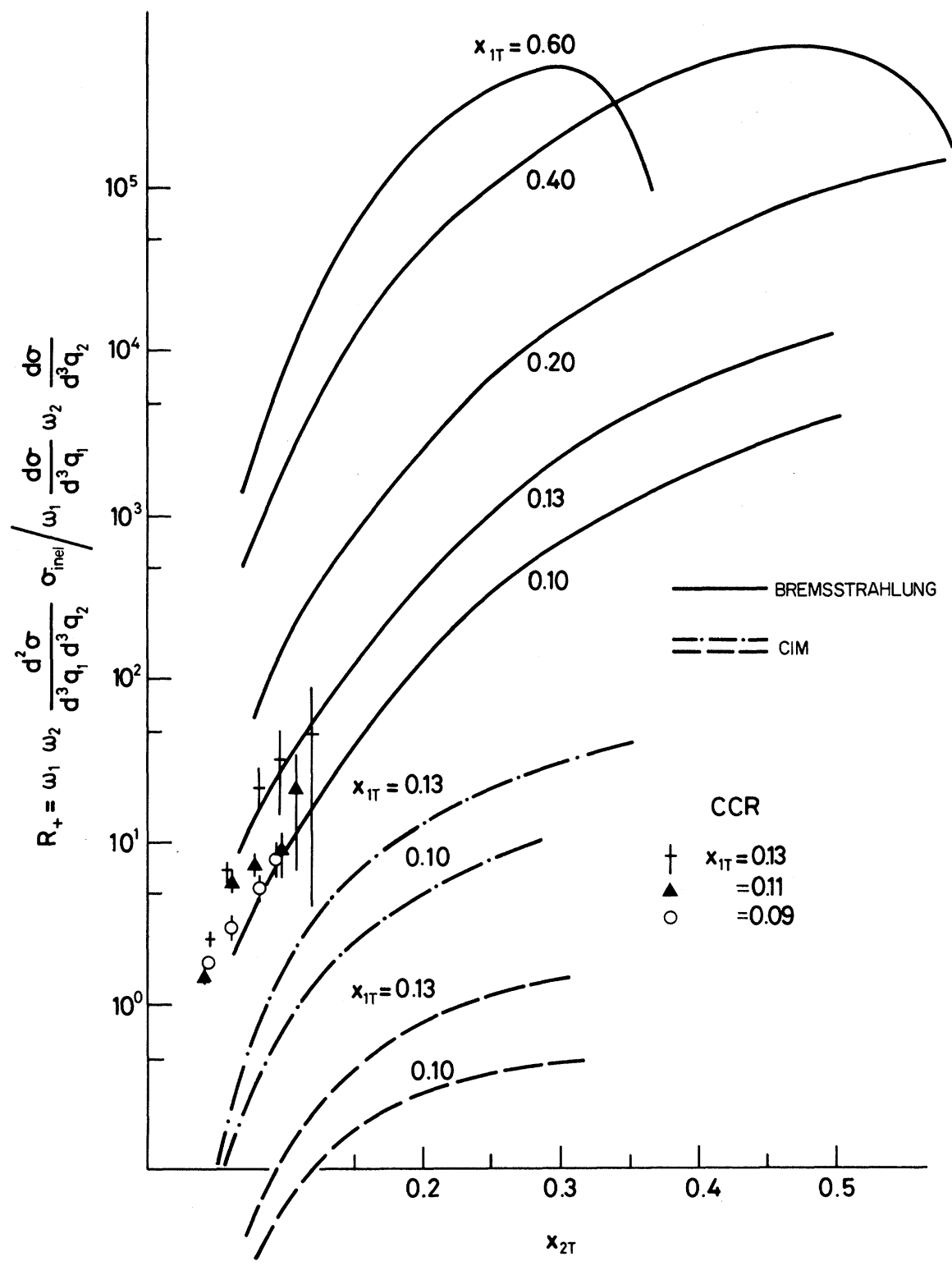

FIG. 6. The correlation coefficient $R_{+}$for two $\pi^{0} \mathrm{~s}$ at $90^{\circ}$ in the same direction. Predictions: solid lines are from bremsstrahlung; dash-dotted lines are from CIM with two-meson probability function $G\left(y_{1}, y_{2}\right)=\left(1-y_{1}\right)\left(1-y_{2}\right) / y_{1} y_{2}($ see Ref. 10 for details); dashed lines are from CIM with $G\left(y_{1}, y_{2}\right)=\left(1-y_{1}-y_{2}\right) /\left(y_{1}+y_{2}\right)$. Data from Ref. 6 . 
where the two mesons $m_{1}, m_{2}$ are produced at $90^{\circ}$ in the c.m. of the colliding protons, in the same direction. Starting from (2.1) it is easy to see that also in the present case the inclusive cross section will be given by $(4.3)$ with only $\overrightarrow{\mathrm{q}}_{2}--\overrightarrow{\mathrm{q}}_{2}$.

We begin again by considering $q_{i T}, i=1,2$, in the range $M^{2} \ll 4 q_{i T}{ }^{2} \ll s$, and we make the replacement (4.4) together with $\left(4.4^{\prime}\right)$, so that an expression like (4.5) arises. The leading term in $V^{2}\left(q_{1}\right) V^{2}\left(q_{2}\right)$ corresponds to $\hat{p}_{3} \simeq \hat{q}_{1} \simeq \hat{q}_{2}$ [Fig. $4(\mathrm{~b})$ ]. We set $\left|\overrightarrow{\mathrm{p}}_{3}\right|=\alpha_{1} q_{1 T}=\alpha_{2} q_{2 T}$ and finally obtain

$$
\begin{aligned}
\omega_{1} \omega_{2} \frac{d^{2} \sigma}{d^{3} q_{1} d^{3} q_{2}} \approx & \frac{1}{2} B \frac{q_{1 T}+q_{2 T}}{\left(q_{1 T} q_{2 T}\right)^{9-G / 2}} V^{2}\left(\alpha_{0}\left(q_{2 T} / q_{1 T}\right)^{1 / 2}\right) \\
& \times V^{2}\left(\alpha_{0}\left(q_{1 T} / q_{2 T}\right)^{1 / 2}\right)
\end{aligned}
$$

$B$ is the same constant as in (4.8). The extra factor of $\frac{1}{2}$ is due to the fact that now $\hat{q}_{1}$ and $\hat{q}_{2}$ are in the same direction [Fig. 4(b)], hence the $d \Omega_{3}$ integration covers only one angle, of opening $\Delta \theta_{3} \sim \frac{1}{2}\left[\left(m / q_{1 T}\right)+\left(m / q_{2 T}\right)\right]$.

The extension to the limit $x_{1 T}+x_{2 T} \rightarrow 1$ is made as before. The factor $\phi\left(q_{1 T}, q_{2}, s\right)$ has the same expression, except $q_{2 T} \rightarrow-q_{2 T}$. Thus, instead of (4.9) we obtain

$$
\phi=1-x_{1 T}-x_{2 T} \equiv \phi_{+}\left(x_{1 T}, x_{2 T}\right) .
$$

The final result is

$$
\begin{aligned}
\omega_{1} \omega_{2} \frac{d^{2} \sigma}{d^{3} q_{1} d^{3} q_{2}} \simeq & \frac{B}{2} \frac{q_{1 T}+q_{2 T}}{\left(q_{1 T} q_{2 T}\right)^{9-G / 2}} V^{2}\left(\alpha_{0}\left(q_{2 T} / q_{1 T}\right)^{1 / 2}\right) \\
& \times V^{2}\left(\alpha_{0}\left(q_{1 T} / q_{2 T}\right)^{1 / 2}\right) \phi_{+}\left(x_{1 T}, x_{2 T}\right) .
\end{aligned}
$$

The correlation coefficient $R_{+}$for two sameside hadrons is defined again by (4.13). Thus we find

$$
\begin{aligned}
R_{+}= & \frac{C}{2}\left(q_{1 T}+q_{2 T}\right)\left(q_{1 T} q_{2 T}\right)^{8-G / 2} \\
& \times \sigma_{\text {inel }} \frac{V^{2}\left(\alpha_{0}\left(q_{2 T} / q_{1 T}\right)^{1 / 2}\right) V^{2}\left(\alpha_{0}\left(q_{1 T} / q_{2 T}\right)^{1 / 2}\right)}{V^{4}\left(\alpha_{0}\right)} \\
& \times \frac{\phi_{+}\left(x_{1 T}, x_{2 T}\right)}{\left(1-x_{1 T}\right)\left(1-x_{2 T}\right)}
\end{aligned}
$$

or approximately

$$
\begin{aligned}
R_{+} \approx & \frac{C}{2}\left(q_{1 T}+q_{2 T}\right)\left(q_{1 T} q_{2 T}\right)^{8-G / 2} \\
& \times \sigma_{\text {inel }} \frac{1-x_{1 T}-x_{2 T}}{\left(1-x_{1 T}\right)\left(1-x_{2 T}\right)} .
\end{aligned}
$$

Notice that $C$ is the same constant as in (4.14) and (4.15), i.e., $R_{+}$is predicted in shape and in magnitude. In Fig. 6 (full lines) we compare the more exact expression (5.5) with the data of the CCR collaboration. Agreement is good.
As we said [fact (b) of Introduction], the main feature of these data is that at least for $x_{1 T} \approx x_{2 T} \approx 0.1$ (i.e., $q_{1 T} \simeq q_{2 T} \simeq 2.6 \mathrm{GeV}$ ) $R_{+}$is $n o t$ much smaller than $R_{\text {.. }}$. This aspect is consistent with the experimental fact (c) of the Introduction (strong correlations between a $\pi^{0}$ of $q_{T}>3 \mathrm{GeV}$ and a charged hadron $h^{ \pm}$of large $q_{T}$ ). All this is in agreement with (5.5) or (5.6), which imply that at those values of $q_{1 T}, q_{2 T}$ we have $R_{+}$somewhat smaller than $R_{\text {_ }}$ but certainly non-negligible. Notice, however, that as $x_{1 T}+x_{2 T} \rightarrow 1$ the ratio $R_{+} / R_{-}$decreases and tends to zero [see (4.14), (5.5) or Figs. 5 and 6].

For comparison, in Fig. 6 (dashed and dashdotted lines) we also present typical predictions of the CIM for $R_{+}$, as calculated in Ref. $10{ }^{21}$ Clearly, $R_{+}$is too small by $1-2$ orders of magnitude. As we discussed in Sec. III alongside a large- $q_{T}$ trigger, the CIM predicts very small probability for observing another large- $q_{T}$ hadron (or for observing a jet). Notice that in the $\mathrm{CIM}^{2}$ this conclusion remains unaffected even if the large $-q_{T}$ hadrons originate from some resonance. ${ }^{10}$

In contrast we have seen that if the underlying mechanism is hadronic bremsstrahlung, some jet is predicted alongside the trigger. This jet may consist of either several slow particles (mainly pions) or a few fast ones. Thus strong correlations for two same-side hadrons of large $q_{T}$ are not unexpected. ${ }^{22}$

\section{SUMMARY OF EXPERIMENTAL TESTS AND CONCLUDING REMARKS}

For convenience we summarize here some characteristic predictions and experimental tests of the bremsstrahlung model. We consider a meson (e.g., pion) trigger of $q_{T} \gtrsim 3 \mathrm{GeV}$ at an angle near $\theta=90^{\circ}$ in the c.m. of the colliding protons:

(a) Alongside and on the opposite side of the trigger there should be two nucleons (more generally baryons) of momenta $\gtrsim 1 \mathrm{GeV}$; there should be no nucleon at small angle. Observation of a nucleon with, say, $x_{L} \equiv 2 p_{L} / \sqrt{s} \gtrsim 0.5$ will be in support of parton models. ${ }^{20}$

(b) Alongside the trigger there should be either some meson jet (weaker than the opposite-side jet) or 1-2 other fast mesons. Thus, for moderate values of $x_{T}$ the same-side CC should be non-negligible, although smaller by a factor of 3-5 than the opposite-side CC.

The above predictions and tests, which have been deduced in our hadronic bremsstrahlung formalism, are also suggested by simple analogy with conventional photon bremsstrahlung. We feel strongly, however, that a better mathematical formalism is necessary. To enumerate some 
reasons, our basic formulas can be rigorously justified only in the soft-neutral-vector-meson approximation; the extension to moderate- and large $-x_{T}$ values (end of Sec. II) is, strictly speaking, arbitrary (its best justification is the reasonableness of the final expressions); the replacement (2.5) of Refs. 3 and 12, which is consistently carried out throughout our work, is dictated only by the inability to calculate fourdimensional integrals like $\int d^{4} x e^{i x \cdot(P-q)} e^{K(x)}$; finally, the form (2.10) of the off-shell "elastic" amplitude $M_{0}$ has only some phenomenological justification. ${ }^{4}$

However, the reasonableness of our results (compared with classical bremsstrahlung) suggests that several of them may survive a more rigorous formulation of the model.

\section{ACKNOWLEDGMENTS}

We would like to thank H. Fried, M. Jacob, Professor R. Hagedorn, and R. Savit for many helpful discussions, and members of the CCRS and ACHM collaborations, especially L. Di Lella, P. Darriulat, and Th. Modis for very useful discussions and for communicating to us their data before publication. One of us (A. P. C.) would like to warmly thank Professor S. Fubini and Professor D. Amati for the hospitality extended to him in the CERN Theory Division, and Professor R. Omnès and Professor B. d'Espagnat for their hospitality at the Laboratoire de Physique Théorique et Particules Elémentaires, Orsay, during his sabbatical leave from McGill University.

\section{APPENDIX}

We would like to consider here relations of the form (2.6), which have been used throughout our work.

We begin with inclusive proton production $p_{1}+p_{2} \rightarrow p_{3}+X$. In this case $^{3,4,12}$

$$
\left\langle\sum_{i} \overrightarrow{\mathrm{q}}_{i}\right\rangle=0 \text {, }
$$

where $\vec{q}_{i}$ are the momenta of the unobserved (vector) mesons emitted in the process. (A1) is plausible by the random nature of the bremsstrahlung emission, but because of its importance we shall provide a derivation.

We denote

$$
\begin{aligned}
\Delta \sigma_{n} \equiv & \frac{M^{4}}{E_{1} E_{2} v_{12}} \frac{\left|M_{0}\right|^{2}}{4 \pi^{2}}\left[\frac{g^{2}}{(2 \pi)^{3}}\right]^{n} \\
& \times \prod_{i=1}^{n} \int \frac{d^{3} q_{i}}{2 \omega_{i}} V^{2}\left(q_{i}\right) \delta^{4}\left(P-\sum_{i=1}^{n} q_{i}\right),
\end{aligned}
$$

where $P=p_{1}+p_{2}-p_{3}-p_{4}$ (Sec. II); then $\left\langle\sum_{i} \overrightarrow{\mathrm{q}}_{i}\right\rangle$ is defined by

$$
\left\langle\sum_{i} \overrightarrow{\mathrm{q}}_{i}\right\rangle=\frac{\int \frac{d^{3} p_{3}}{E_{3}} \int \frac{d^{3} p_{4}}{E_{4}} \sum_{n}\left(\overrightarrow{\mathrm{q}}_{1}+\overrightarrow{\mathrm{q}}_{2}+\cdots+\overrightarrow{\mathrm{q}}_{n}\right) \Delta \sigma_{n}}{\int \frac{d^{3} p_{3}}{E_{3}} \int \frac{d^{3} p_{4}}{E_{4}} \sum_{n} \Delta \sigma_{n}} .
$$

In the c.m. of the colliding protons

$$
\begin{aligned}
\delta^{4}\left(P-\sum_{i=1}^{n} q_{i}\right)= & \delta\left(\sqrt{s}-E_{3}-E_{4}-\mathcal{E}_{n}\right) \\
& \times \delta^{3}\left(\overrightarrow{\mathrm{p}}_{3}+\overrightarrow{\mathrm{p}}_{4}+\sum_{i=1}^{n} \overrightarrow{\mathrm{q}}_{i}\right)
\end{aligned}
$$

where $\mathcal{E}_{n}$ is the total energy of $n$ mesons; thus for any $n$,

$$
\overrightarrow{\mathrm{q}}_{1}+\overrightarrow{\mathrm{q}}_{2}+\cdots \cdot \overrightarrow{\mathrm{q}}_{n}=-\overrightarrow{\mathrm{p}}_{3}-\overrightarrow{\mathrm{p}}_{4}
$$

and (A3) becomes

$$
\left\langle\sum_{i} \overrightarrow{\mathrm{q}}_{i}\right\rangle=\frac{\int \frac{d^{3} p_{3}}{E_{3}} \int \frac{d^{3} p_{4}}{E_{4}}\left(-\overrightarrow{\mathrm{p}}_{3}-\overrightarrow{\mathrm{p}}_{4}\right) \sum_{n} \Delta \sigma_{n}}{\int \frac{d^{3} p_{3}}{E_{3}} \int \frac{d^{3} p_{4}}{E_{4}} \sum_{n} \Delta \sigma_{n}} .
$$

We now consider two different kinematic configurations: a first one corresponding to momenta $\overrightarrow{\mathrm{p}}_{3}, \overrightarrow{\mathrm{p}}_{4}, \vec{q}_{i}$, and a second one corresponding to $\overrightarrow{\mathrm{p}}_{3}^{\prime}=-\overrightarrow{\mathrm{p}}_{3}, \overrightarrow{\mathrm{p}}_{4}^{\prime}=-\overrightarrow{\mathrm{p}}_{4}, \overrightarrow{\mathrm{q}}_{i}^{\prime}=-\overrightarrow{\mathrm{q}}_{i}$, and compare the integrands of (A6). The function $V^{2}(q)$ contains scalar products of the form $p_{\alpha} \cdot q$ and $p_{\alpha} \cdot p_{\beta}$. In the c.m. of the colliding protons we easily find $\left(q, q^{\prime}\right.$ stand for any of $q_{i}, q_{i}^{\prime}$ )

$$
\begin{array}{ll}
p_{1} \cdot q^{\prime}=p_{2} \cdot q, & p_{2} \cdot q^{\prime}=p_{1} \cdot q, \\
p_{3}^{\prime} \cdot q^{\prime}=p_{3} \cdot q, & p_{4}^{\prime} \cdot q^{\prime}=p_{4} \cdot q, \\
p_{1} \cdot p_{3}^{\prime}=p_{2} \cdot p_{3}, & p_{1} \cdot p_{4}^{\prime}=p_{2} \cdot p_{4}, \\
p_{2} \cdot p_{3}^{\prime}=p_{1} \cdot p_{3}, & p_{2} \cdot p_{4}^{\prime}=p_{1} \cdot p_{4},
\end{array}
$$

etc. We easily deduce

$$
V^{2}\left(q^{\prime}\right)=V^{2}(q) \text {. }
$$

We further assume that $\left|M_{0}\left(p_{1}, p_{2}, p_{3}^{\prime}, p_{4}^{\prime}\right)\right|$ $=\left|M_{0}\left(p_{1}, p_{2}, p_{3}, p_{4}\right)\right|$, which is certainly satisfied by the "elastic" amplitudes (2.10). The symmetry of the $d^{3} p_{3}, d^{3} p_{4}$, and $d^{3} q_{i}$ integrations easily implies (A1).

Next we consider $p_{1}+p_{2} \rightarrow m+\boldsymbol{X}$ when the meson $m$ is produced at large angles with momentum $|\overrightarrow{\mathrm{q}}|$ satisfying $M^{2} \ll 4|\overrightarrow{\mathrm{q}}|^{2} \ll s$ [range (2.4)]. In our model $m$ is one of the mesons accompanying the nucleon $p_{3}$ (Sec. II); thus we expect the relation (A1) to take the form (2.6). Similar considerations apply to $p_{1}+p_{2} \rightarrow m_{1}+m_{2}+X$ [when the mesons $m_{i}(i=1,2)$ are produced at large angles with momenta satisfying $\left.M^{2} \ll 4\left|\overrightarrow{\mathrm{q}}_{i}\right|^{2} \ll s\right]$; then we obtain $\left(4.4^{\prime}\right)$. 
*On sabbatical leave from McGill University, Montreal, Quebec, Canada.

${ }^{1} \mathrm{P}$. V. Landshoff, in Proceedings of the XVII International Conference on High Energy Physics, London, 1974, edited by J. R. Smith (Rutherford Laboratory, Chilton, Didcot, Berkshire, England, 1974), p. V-57.

${ }^{2}$ R. Blankenbecler and S. J. Brodsky, Phys. Rev. D 10, 2973 (1974); in Proceedings of SLAC Summer Institute on Particle Physics, 1974, SLAC Report No. SLAC-179 (unpublished), Vol. I, p. 361.

${ }^{3}$ H. M. Fried and T. K. Gaisser, Phys. Rev. D 7,741 (1973); H. M. Fried, contribution to the XVII International Conference on High-Energy Physics, London, 1974 (unpublished).

${ }^{4}$ A. P. Contogouris, J. P. Holden, and E. N. Argyres, Phys. Lett. 51B, 251 (1974); Nucl. Phys. B85, 102 (1975).

${ }^{5} \mathrm{~F}$. W. Büsser et al., CERN-Columbia-Rockefeller (CCR) Collaboration, Phys. Lett. 51B, 306 (1974); Pisa-Stony Brook Collaboration, CERN report, 1974 (unpublished); B. Alper et al., Lett. Nuovo Cimento 11, 173 (1974).

${ }^{6} \mathrm{~F}$. W. Büsser et al. (CCR Collaboration), Phys. Lett. 51B, 311 (1974).

${ }^{7}$ M. Banner, CERN-Columbia-Rockefeller-Saclay (CCRS) Collaboration, in Proceedings of the $X$ Rencontre de Moriond, Meribel-les-Allues, 1975, edited by J. Tran Thanh Van (Université de ParisSud, Orsay, 1975).

${ }^{8} \mathrm{P}$. Darriulat, Aachen-CERN-Heidelberg-Munich (ACHM) Collaboration, in Proceedings of the $X$ Rencontre de Moriond, Meribel-les-Allues, 1975, edited by "J. Tran Thanh Van (Ref. 7); Th. Modis, private communication; also see the work of the Pisa-Stony Brook Collaboration, reported in Ref. 1.

${ }^{9} \mathrm{M}$. Jacob, in proceedings of the CERN ISR Discussion Meeting, CERN report (unpublished).

${ }^{10}$ D. Sciff, A. P. Contogouris, and J. Alonso, Phys. Lett. 55B, 87 (1975).

${ }^{11}$ S. M. Berman, J. D. Bjorken, and J. B. Kogut, Phys. Rev. D 4, 3388 (1971); S. D. Ellis and M. B. Kisslinger, ibid. 9 , 2027 (1974).

${ }^{12}$ H. M. Fried and T. K. Gaisser, Phys. Rev. D 6,2560 (1972).

${ }^{13}$ M. Islam and J. Owers, Jr., Phys. Rev. D $\underline{7}, 3784$ (1974).

${ }^{14}$ The exact form of $(2.2)$ is

$$
\begin{aligned}
\tilde{V}(q)= & \frac{2 p_{1}-q}{q^{2}-2 p_{1} \cdot q}+\frac{2 p_{2}-q}{q^{2}-2 p_{2} \cdot q}+\frac{2 p_{3}+q}{q^{2}+2 p_{3} \cdot q} \\
& +\frac{2 p_{4}+q}{q^{2}+2 p_{4} \cdot q} .
\end{aligned}
$$

However, the amplitude for the process $p_{1}+p_{2}$ $\rightarrow p_{3}+p_{4}+n m$, representing emission of $n$ neutral vector mesons, is $M_{n}=g^{n} M_{0} \Pi_{i=1}^{n} V\left(q_{i}\right) \cdot \epsilon_{i}$, where $\epsilon_{i}$ $=$ polarization four-vector for meson $i$. Thus, after insertion of $\tilde{V}\left(q_{i}\right)$ owing to the condition $q_{i} \cdot \epsilon_{i}=0$, the terms $\mp q_{i}$ in the numerator cancel.

${ }^{15}$ The precise form of the exponent $G$, in particular as a function of $\ln s$, depends on the method of calculation of $K(0)$ (Refs. 3,4). What we consider of importance is that for reasonable values of the coupling $g$ we obtain $G=9-10$.

${ }^{16}$ H. A. Gordon, M. M. Habibi, Kwan-Wu Lai, and I. Stumer, Phys. Rev. Lett. 34, 284 (1975); J. M. Gaillard, in Proceedings of the $X$ Rencontre de Moriond, Meribel-les-Allues, 1975, edited by J. Tran Thanh Van (Ref. 7).

${ }^{17} \mathrm{~J}$. D. Bjorken, in proceedings of the Second International Conference on Elementary Particles, Aix-enProvence, 1973 [J. Phys. (Paris) Suppl. 34, C1-385 (1973)].

${ }^{18}$ The two jets along $\hat{p}_{1}$ and $\hat{p}_{2}=-\hat{p}_{1}$ will produce a distribution of mesons symmetric about $\eta=0$. Some of these mesons will be mixed with those of the jets along $p_{1}$ and $p_{2}$; and the experimenter will see the last two jets somewhat shifted towards $\eta=0$, as in Fig. 2 (c).

${ }^{19}$ We would like to thank members of the ACHM Collaboration for pointing out this simple test.

${ }^{20} \mathrm{We}$ would like to thank M. Jacob for stressing this point.

${ }^{21}$ In plotting the predictions of CIM for $R_{+}$(Fig. 6) we have multiplied the results of Ref. 10 by factor of 3 . This extra factor of $R_{+}\left(\pi^{0}-\pi^{0}\right.$ same) versus $R_{-}$ $\left(\pi^{0}-\pi^{0}\right.$ opposite) follows by counting quark-antiquark states. We thank Bob Savit for stressing this point to us.

${ }^{22}$ In both Secs. IV and V $\left|M_{0}\right|$ is taken to have the same expression, Eq. (2.10). This is well-justified in the soft-meson approximation, and may also be reasonable when $M^{2}<<4 q_{i T}^{2}<s, i=1,2$. Also, it is implicitly related to the presence of two jets in bremsstrahlung. In contrast, the CIM inclusive cross sections contain integrals over the differential cross section $d \sigma / d \hat{t}$ of the appropriate subprocesses (Refs. 2, 10), and in the cases of interest $d \sigma / d \hat{t} \sim \hat{s}^{-4}$, where $\hat{s}$ $=$ c.m. energy of subprocess (Ref. 10). For two sameside hadrons $\hat{s}$ is much greater than for two oppositeside hadrons; thus in the former case $d \sigma / d \hat{t}$ is very small (Ref. 10). 